\title{
A General Radiation Model for Sound Fields and Nearfield Acoustical Holography in Wedge Propagation Spaces
}

\author{
Falk-Martin Hoffmann* and Filippo Maria Fazi \\ Institute of Sound and Vibration Research, University of Southampton, Southampton, Hampshire, SO17 1BJ, UK \\ Earl G. Williams \\ Naval Research Laboratory, Code 7106, Washington, DC 20375, USA \\ Simone Fontana \\ Huawei European Research Center, Riesstrasse 25 C3.0G, 80992 Munich, Germany
}

(Dated: September 13, 2017)

\begin{abstract}
In this work an expression for the solution of the Helmholtz equation for wedge spaces is derived. Such propagation spaces represent scenarios for many acoustical problems where a free field assumption is not eligible. The proposed sound field model is derived from the general solution of the wave equation in cylindrical coordinates, using sets of orthonormal basis functions. The latter are modified to satisfy several boundary conditions representing the reflective behaviour of wedge-shaped propagation spaces. This formulation is then used in the context of Nearfield Acoustical Holography (NAH) and to obtain the expression of the Neumann Green function. The model and its suitability for NAH is demonstrated through both numerical simulations and measured data, where the latter was acquired for the specific case of a loudspeaker on a hemi-cylindrical rigid baffle.

PACS numbers: *43.35.Sx, $* 43.60 . \mathrm{Sx}, * 43.20 .-\mathrm{f}, 43.20 .+\mathrm{g}$

Keywords: Wedges Spaces; Holography; Boundary Conditions; Neumann Green Function
\end{abstract}

\section{INTRODUCTION}

Analytical sound field models exist in a variety of mathematical forms that can differ to suit different types of acoustic environments. Most rigorous analytical models are solutions to the acoustic wave equation (at least within a problem-specific region of validity). Some models are simply based on a specific coordinate system that best describes a given problem. Some more specific models employ boundary conditions to include diffraction of scattering objects within the sound field. However, adding boundary conditions to a problem quickly makes it difficult to formulate an analytical model, which is where numerical methods can be applied instead. Especially models based on functional analysis are widely used for both sound field capture/analysis [1-9], reproduction/control [10-14] and Nearfield Acoustical Holography $(\mathrm{NAH})[15-20]$.

In the absence of scattering objects, a typical approach to modelling a specific acoustic scenario is to take the solution pertaining to the free and undisturbed field (within a bounded volume) in the most suitable coordinate system for the given problem. All sound sources are assumed to be located outside of a bounded volume of interest and modelled on the basis of the superposition of either plane waves or point sources $[2,21,22]$. These solutions may be sufficient for many acoustic problems, yet those where scattering has a significant impact on the sound field require more accurate solutions. For example, the sound

\footnotetext{
*F.Hoffmann@soton.ac.uk; Corresponding author.
}

field models for acoustic transducer arrays often need to be modified to account for the scattering due to the array's own physical presence in the sound field. This is achieved by superimposing the specific solution for the sound field radiated from a source distribution to the free field solution, so that the sum satisfies the boundary condition $[2-5,8,23,24]$.

The example of the model for the sound field of acoustic transducer arrays shows how introducing boundary conditions to a problem can serve to better describe the characteristics of a specific acoustic environment. Further examples for acoustic environments that require several boundary conditions are ducts and transmission lines $[25,26]$, rooms $[27,28]$ and underwater shorelines $[1,9,29]$. Asvestas et al. [30] described the wave equation as separable inside infinite wedges for both the Dirichlet and the Neumann boundary condition, yielding analytical expressions for the field of plane waves, and point and line sources through an infinite series expansion and infinite integration in the wavenumber domain. Buckingham $[1,29]$ adopted their point source model to the field of shoreline underwater acoustics for different boundary conditions on the faces of the wedge. Luo and Zhang [9] recently published exact solutions for both the Dirichlet and Neumann boundary conditions in the infinite wedge, including a numerical approach to integrate over potentially occurring singularities.

Boundary conditions also play a role in the application of Rayleigh's First Integral formula to describe the radiation from a piston integrated into an infinite baffle $[26,28]$. While this formula is a well established tool to model the radiation from panels, loudspeaker drivers or similar structures, it is still derived for a practically 
non-existent environment. Williams [2] introduced the concept of Rayleigh-like formulae to transfer the principle to other coordinate systems and geometries.

In principle, these Rayleigh-like formulae project the behaviour of one specific boundary to any other point in space. NAH extends this concept to link two boundaries within a given sound field through propagators, which makes NAH particularly well suited for the use with coordinate systems in which the acoustic wave equation is separable [2, 16, 31, 32]. Their solutions enable propagation of holograms either further away from, or back towards their source. These forward or inverse problems, respectively, can be solved through either an eigenvalue decomposition or a singular value decomposition of the solutions of the wave equation. Williams et al. presented an overview for planar and cylindrical NAH in [31]. Other notable NAH (related) methods can be found in [33-39], e.g. ESM, IBEM, HELS and SONAH. One prominent application of NAH is to identify the vibration pattern on the surface of an object [2, 31, 32], which makes it conceptually the inverse of Rayleigh-like formulae. Another application is to investigate the directivity of sound sources by looking at the pressure field directly $[15,40]$ or by evaluating the intensity field from the reconstructed pressure and velocity holograms [18].

One key issue with NAH is that inverse problems are known to suffer from ill-conditioning when very small eigen/singular values meet measurement noise [31, 41, 42]. In that case, regularisation of the inverse can yield a stable solution, which may come at the cost of spatial resolution. However, regularisation techniques based on sparsity assumptions and statistical optimisation have been proposed recently $[39,43,44]$ that have the potential of overcoming this issue.

This work introduces a radiation model for propagation spaces that are relevant for many practical acoustical applications, e.g. the measurement of the radiation from a loudspeaker (or any other acoustic source) in the corner of a room or similar environments. It adopts the solution of the wave equation in acoustical wedges by Asvestas et al. [30] as the basic model and extends it by yet another boundary condition. The final model enables the deduction of a Neumann-Green Function (NGF) and the formulation of a Rayleigh-like formula. Both provide tools to describe the acoustic radiation from sources in rooms or other wedge-shaped propagation spaces, e.g. a corner between two walls. Furthermore, it is shown that the model can be used for NAH. The findings are confirmed through both simulated and measured data.

The remainder of this work is organised as follows. Section II introduces the sound field model in the form of the solution to the acoustic Helmholtz equation in infinite wedge propagation spaces. On the basis of that model, a Rayleigh-like formula and an NGF are derived in Section III. Section IV defines the radial functions associated with the combination of the spatial modes, enabling the study of the propagation behaviour of individual wave components. Using these radial functions, the propagators needed for the reconstruction of both velocity and pressure from a measured pressure hologram are defined in Section V. In analogy to the previous three sections, Section VI presents the most important equations and expressions for the finite wedge. Section VII presents simulated sound fields for two elementary types of source, one of which is later used to provide a comparison to measured data. The results of NAH with both simulated holograms and measured holograms obtained from a specially designed measurement rig are presented and discussed in Section VIII before the findings of this work are summarised in the final section.

\section{SOUND FIELD MODEL}

\section{A. Description of the Geometry}

The reference geometry used throughout this paper is based on the cylindrical coordinate system shown in Fig. 1. The propagation space is restricted to an infinite

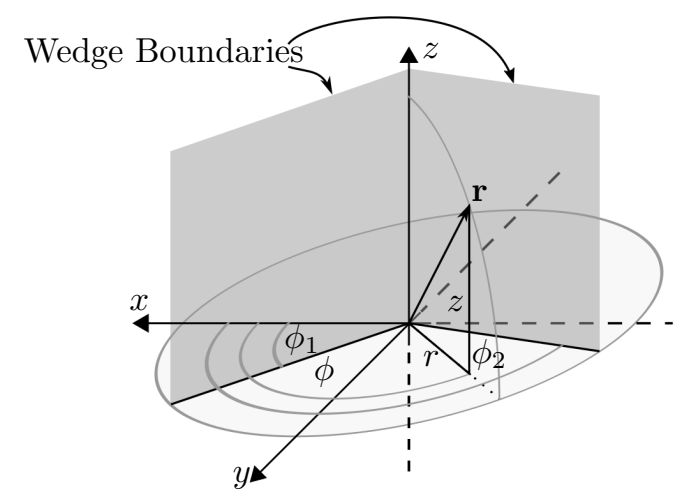

FIG. 1: Cylindrical coordinates with radius $r$, azimuth angle $\phi$ and height $z$ with wedge boundaries at $\phi_{1}$ and $\phi_{2}$.

wedge by introducing two planes, $\phi=\phi_{1}$ and $\phi=\phi_{2}$, with $\phi_{2}>\phi_{1}$ and whose intersection coincides with the $z$-axis (see Fig. 1). The angle between these planes is $\kappa=\phi_{2}-\phi_{1}$. The sound field is defined in the wedge space for which the angular coordinate $\phi \in\left[\phi_{1}, \phi_{2}\right]$. All considerations presented in the following pertain to the frequency domain, and the dependency on $e^{-i \omega t}$ and the parameter $\omega$ are omitted in all expressions for the sake of brevity.

\section{B. General Solution of the Helmholtz Equation in the Infinite Wedge}

The general solution of the Helmholtz Equation in cylindrical coordinates for an infinite wedge can be derived through a separation of variables approach in the 
frequency domain [2], yielding the expression

$$
p(\mathbf{r})=\sum_{n=0}^{\infty} \Phi_{n}(\phi) \int_{-\infty}^{\infty} A_{n}\left(k_{z}\right) R_{n}\left(k_{r} r\right) Z\left(k_{z} z\right) d k_{z},
$$

with $\mathbf{r}=(r, \phi, z)^{T}$.

The angular basis functions $\Phi_{n}(\phi)$ depend on the boundary condition that describes the acoustic properties on the flanks of the wedge at $\phi=\phi_{1}, \phi_{2}$, which can in principle be a Robin boundary condition

$$
\left.a p(r, \phi, z)\right|_{\phi=\phi_{1}, \phi_{2}}+\left.b \frac{\partial p(r, \phi, z)}{\partial \phi}\right|_{\phi=\phi_{1}, \phi_{2}}=g(r, \phi, z),
$$

for $\phi=\phi_{1}, \phi_{2}, a, b \in \mathbb{N}_{0}$, and $g(\phi) \in \mathbb{C}$. The latter specifies the acoustic impedance on the boundary. In this work, only the impedances associated with the pressure release condition $\left.p(\mathbf{r})\right|_{\phi=\phi_{1}, \phi_{2}}=0$ and with the rigid condition $\left.\frac{\partial}{\partial \phi} p(\mathbf{r})\right|_{\phi=\phi_{1}, \phi_{2}}=0$ are considered. The benefit behind this restriction is that either of them leads to a countable Total Orthonormal Set (TOS) [45] of angular basis functions

$$
\Phi_{n}(\phi)=\nu_{n} \begin{cases}\sin \left(\frac{n \pi}{\kappa}\left(\phi-\phi_{1}\right)\right), & (\text { Pressure Release BC) } \\ \cos \left(\frac{n \pi}{\kappa}\left(\phi-\phi_{1}\right)\right), & (\text { Rigid BC) }\end{cases}
$$

with $\nu_{n}=\sqrt{\frac{2-\delta_{n}}{\kappa}}, \delta_{n}$ representing the Kronecker Delta, $n \in \mathbb{N}_{0}$ and $\phi_{1}, \phi_{2} \in[0,2 \pi)$. In either case it holds that the basis functions $\Phi_{n}(\phi)$ are all real-valued and hence describe exclusively standing wave components along the $\phi$-direction.

This choice of $\Phi_{n}(\phi)$ requires resolving Bessel's equation to identify the radial basis functions, leading to solutions in the form of Bessel, Neumann and Hankel functions of potentially non-integer order (compare to [2]). Since this work is devoted to the radiation of sound away from the coordinate origin, given the time convention used in this work, only the Hankel functions of the first kind are considered as radial basis functions, so that

$$
R_{n}\left(k_{r} r\right)=H_{\frac{n \pi}{\kappa}}^{(1)}\left(k_{r} r\right) .
$$

Note that this solution is the same for both boundary conditions. The axial basis functions are defined through

$$
Z\left(k_{z} z\right)=\frac{e^{i k_{z} z}}{\sqrt{2 \pi}}
$$

with the relation between the radial and axial component of the wave number

$$
k_{r}=\sqrt{k^{2}-k_{z}^{2}},
$$

where $k=\frac{\omega}{c}$ represents the wave number as a function of the angular frequency, $\omega$, and the speed of sound, $c$.
The solution in (1) is identical to the one presented in $[1,9,29]$ and the coefficients $A_{n}\left(k_{z}\right)$ describe a pressure field radiated from sources located within a cylinder of radius $r_{S}$ around the origin [2]. In that case, Eq. (1) is only valid for $r>r_{S}$. The particle velocity vector field

$$
\mathbf{v}(\mathbf{r})=v_{r}(\mathbf{r}) \cdot \mathbf{e}_{r}+v_{\phi}(\mathbf{r}) \cdot \mathbf{e}_{\phi}+v_{z}(\mathbf{r}) \cdot \mathbf{e}_{z},
$$

in the propagation space can be obtained from the pressure model by exploiting Euler's equation in the frequency domain, $i \rho_{0} c k \vec{v}(\mathbf{r})=\nabla p(\mathbf{r})$.

In preparation for the acoustical holography application of the model, the spatial spectra corresponding to the pressure and the particle velocity are introduced in the next subsection.

\section{Pressure and Velocity Spectrum}

Let $\{p(\mathbf{r})\}_{\mathbf{r} \in \Lambda}$ be a hologram of the pressure at a given radius $r_{H}$ with

$$
\Lambda:=\left\{\mathbf{r}: r=r_{H}, \phi \in\left[\phi_{1}, \phi_{2}\right], z \in \mathbb{R}\right\} .
$$

Taking the scalar product $[21,45]$ of the hologram and the model's basis functions defines the spatial analysis transform, the result of which provides the holograms' spectrum through

$$
P_{n}\left(k_{z}, r_{H}\right)=\int_{\phi_{1}}^{\phi_{2}} \int_{-\infty}^{\infty} p\left(r_{H}, \phi, z\right) \Phi_{n}(\phi) Z^{*}\left(k_{z} z\right) d z d \phi .
$$

Exploiting the orthonormality relation of TOSs to invert the expressions in (9) provides the corresponding spatial synthesis transform

$$
p\left(r_{H}, \phi, z\right)=\sum_{n=0}^{\infty} \int_{-\infty}^{\infty} P_{n}\left(k_{z}, r_{H}\right) \Phi_{n}(\phi) Z\left(k_{z} z\right) d k_{z} .
$$

Replacing the expression for the pressure in Eq. (9) by the expression in (1), and exploiting the orthonormality relation of orthonormal sets $[21,45]$ yields the relation between the sound field coefficients and the pressure spectrum

$$
A_{n}\left(k_{z}\right) R_{n}\left(k_{r} r_{H}\right)=P_{n}\left(k_{z}, r_{H}\right) .
$$

This equation can be rewritten for $A_{n}\left(k_{z}\right)$, if $\left|R_{n}\left(k_{r} r_{H}\right)\right| \neq 0$, which applies for the Hankel functions of the first kind for $0<\left|\alpha r_{H}\right|<\infty$ and $r_{H} \in \mathbb{R}$, where $\alpha=k_{r}$ is defined in Eq. (6) [46].

In accordance with what is shown for the pressure hologram above, the expression for the spectrum of a particle velocity hologram is given by

$$
\mathbf{V}_{n}\left(k_{z}, r_{H}\right)=\int_{\phi_{1}}^{\phi_{2}} \int_{-\infty}^{\infty} \mathbf{v}\left(r_{H}, \phi, z\right) \Phi_{n}(\phi) Z^{*}\left(k_{z} z\right) d z d \phi .
$$


Since $\mathbf{v}(\mathbf{r})$ is a vector, it follows that the resulting spectrum must have the same dimensions.

At this point, the model for sound propagation in infinite wedges is complete, however, some applications can benefit from alternative formulations. The following section presents the derivation of Rayleigh-like formula [2] and NGF on the basis of the developed model.

\section{RAYLEIGH-LIKE FORMULA AND NEUMANN-GREEN FUNCTION FOR INFINITE WEDGES}

Rayleigh-like formulae describe the pressure field radiated from the velocity distribution on a given rigid boundary

$$
V:=\left\{\mathbf{r}: r=r_{S}, \phi \in\left[\phi_{1}, \phi_{2}\right], z \in \mathbb{R}\right\}
$$

inside the propagation space and are thus conceptually similar to Rayleigh's first integral formula [2]. Since the velocity at $V$ causes acoustic radiation, the entire boundary effectively becomes a source. The developed model already supports this premise through the choice of the radial basis functions in (4). It can be assumed that the velocity of $V$ is limited to a radial component, i.e. $\mathbf{v}\left(r_{S}, \phi, z\right)=v_{r}\left(r_{S}, \phi, z\right) \cdot \mathbf{e}_{r}$ (see Fig. 2) [2]. The nature of the velocity $\mathbf{v}\left(r_{S}, \phi, z\right)$ implies that

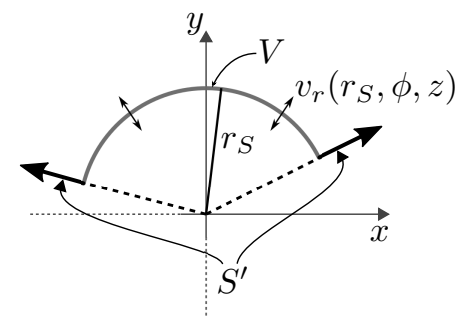

FIG. 2: Concept of the radiation from a curved boundary $V$ with the given velocity profile $v_{r}\left(r_{S}, \phi, z\right)$ inside the wedge defined by the rigid boundaries $S^{\prime}$.

$\mathbf{V}_{n}\left(k_{z}, r_{s}\right)=V_{n}\left(k_{z}, r_{S}\right) \cdot \mathbf{e}_{r}$. In this case, Euler's equation reduces to

$$
V_{n}\left(k_{z}, r\right)=\frac{-i}{\rho_{0} c k} \frac{\partial}{\partial r} P_{n}\left(k_{z}, r\right)
$$

Since the coefficients $A_{n}\left(k_{z}\right)$ fully describe a sound field inside the infinite wedge, it can be derived from Eq. (11) that the relation between two pressure spectra at different radii is

$$
P_{n}\left(k_{z}, r\right)=\frac{R_{n}\left(k_{r} r\right)}{R_{n}\left(k_{r} r_{S}\right)} P_{n}\left(k_{z}, r_{S}\right) .
$$

This result is conceptually analogous to NAH in other geometries, e.g. spherical and cylindrical [2]. Using the above to replace $P_{n}\left(k_{z}, r\right)$ in (14) yields

$$
V_{n}\left(k_{z}, r\right)=\frac{-i k_{r}}{\rho_{0} c k} \frac{R_{n}^{\prime}\left(k_{r} r\right)}{R_{n}\left(k_{r} r_{S}\right)} P_{n}\left(k_{z}, r_{S}\right)
$$

where $R_{n, \kappa}^{\prime}(x)=\frac{\partial}{\partial x} H_{\frac{n \pi}{\kappa}}^{(1)}(x)$. Provided that $k_{r} R_{n}^{\prime}\left(k_{r} r\right) \neq$ 0, transposing Eq. (16) for the pressure spectrum $P_{n}\left(k_{z}, r\right)$ and exchanging $r$ with $r_{S}$ yields

$$
P_{n}\left(k_{z}, r\right)=i \rho_{0} c k \frac{R_{n}\left(k_{r} r\right)}{k_{r} R_{n}^{\prime}\left(k_{r} r_{S}\right)} V_{n}\left(k_{z}, r_{S}\right), r \geq r_{S} .
$$

The synthesis of the pressure $p(\mathbf{r})$ at radius $r$ from the result in (17) through evaluation of Eq. (10) leads to the expression for the pressure as a function of the velocity spectrum on the boundary $V$

$$
\begin{gathered}
p(\mathbf{r})=i \rho_{0} c k \sum_{n=0}^{\infty} \int_{-\infty}^{\infty} \frac{R_{n}\left(k_{r} r\right)}{k_{r} R_{n}^{\prime}\left(k_{r} r_{S}\right)} V_{n}\left(k_{z}, r_{S}\right) \\
\cdot \Phi_{n}(\phi) Z\left(k_{z} z\right) d k_{z} .
\end{gathered}
$$

An analytic expression for (18) is hard to find due to the rather complex integral. Therefore it is common to limit the bounds of integration and solve it numerically instead. When attempting that, it is important to deal with the case when $k_{r}=0$ as it poses a singularity. To solve that issue, Luo and Zhang proposed to marginally shift the integration path into the complex plane in order to avoid the singularity [9]. Furthermore, it is necessary to limit the integration bounds for the numerical calculation. The error introduced by this limitation can be controlled by considering the evanescent behaviour of higher order components [2]. This is more thoroughly discussed in Section IV.

Equation (18) can be used to derive both the Rayleighlike formula and the NGF for radiation problems based on the normal velocity distribution $v_{n}$ on a boundary $S=V \cup S^{\prime}$ (see Fig. 2), where

$$
S^{\prime}:=\left\{\mathbf{r}: r>r_{S}, \phi=\left[\phi_{1}, \phi_{2}\right], z \in \mathbb{R}\right\}
$$

The defining condition of an NGF is that it satisfies $\left.\frac{\partial G_{N}}{\partial n}\right|_{S}=0$, where $n$ denotes the geometrical dimension that locally corresponds to the normal vector on $S$. However, this condition is inherent to the flanking walls for the developed sound field model. This entails that the NGF depends solely on the boundary condition

$$
\left.\frac{\partial G_{N}}{\partial r}\right|_{V}=0
$$

Referring to the Kirchhoff Integral Equation (KIE) as given in [2], the Rayleigh-like formula for the infinite wedge is based on the NGF and must be of the form

$$
p(\mathbf{r})=i \rho_{0} c k \int_{\phi_{1}}^{\phi_{2}} \int_{-\infty}^{\infty} G_{N}\left(\mathbf{r} \mid \mathbf{r}^{\prime}\right) v_{n}\left(\mathbf{r}^{\prime}\right) r_{S} d z^{\prime} d \phi^{\prime}
$$


with $\mathbf{r}^{\prime}=\left(r_{S}, \phi^{\prime}, z^{\prime}\right)$. Using (12) to compare the Rayleigh-like formula to the expression in (18) yields the Neumann-Green function for the infinite wedge

$$
\begin{aligned}
G_{N}\left(\mathbf{r} \mid \mathbf{r}^{\prime}\right)=\sum_{n=0}^{\infty} & \int_{-\infty}^{\infty} \frac{1}{r_{S}} \frac{R_{n}\left(k_{r} r\right)}{k_{r} R_{n}^{\prime}\left(k_{r} r_{S}\right)} \\
& \cdot \Phi_{n}\left(\phi^{\prime}\right) Z^{*}\left(k_{z} z^{\prime}\right) \Phi_{n}(\phi) Z\left(k_{z} z\right) d k_{z}
\end{aligned}
$$

To confirm that this results satisfies (20), (22) is derived w.r.t. $r$ and the result evaluated for $\mathbf{r} \in V$, yielding

$$
\begin{array}{r}
\left.\frac{\partial G_{N}\left(\mathbf{r} \mid \mathbf{r}^{\prime}\right)}{\partial r}\right|_{\mathbf{r} \in V}=\sum_{n=0}^{\infty} \int_{-\infty}^{\infty} \frac{1}{r_{S}} \Phi_{n}\left(\phi^{\prime}\right) Z^{*}\left(k_{z} z^{\prime}\right) \\
\cdot \Phi_{n}(\phi) Z\left(k_{z} z\right) d k_{z}
\end{array}
$$

From the completeness relation of total orthonormal sets $[2,45]$, it follows that

$$
\left.\frac{\partial G_{N}\left(\mathbf{r} \mid \mathbf{r}^{\prime}\right)}{\partial r}\right|_{\mathbf{r} \in V}=\frac{1}{r_{S}} \delta\left(\phi-\phi^{\prime}\right) \delta\left(z-z^{\prime}\right) .
$$

This confirms that the normal derivative of the NGF is indeed equal to zero everywhere on the boundary $V$ but at the position of the singularity/point source $\mathbf{r}^{\prime}=\left(r_{S}, \phi^{\prime}, z^{\prime}\right)^{T}$ (compare to Chapter 8 in [2]).

With the Rayleigh-like formula given in explicit form through Eq. (21), it is now possible to describe the radiated sound field from arbitrary source distributions on the boundary $V$.

\section{RADIAL FUNCTIONS}

The practical implementations of Eq. (18) and Eq. (21) require limitation of the integration bounds and truncation of the sum. The following considerations are equally relevant for both the Rayleigh-like formula and the NGF, but their meaning becomes more intuitive when evaluating the expression in (18).

The determining factor for the limitation/truncation is the transfer function of the velocity modes on the boundary to the corresponding pressure modes at a given observation radius $r$. These transfer functions are also referred to as 'radial functions' and can be identified from Eq. (18) as shown in the following subsection.

\section{A. Identifying the Radial Functions}

Considering that both axial and angular basis functions are orthonormal, the radial functions for the infinite wedge are identified as

$$
\Gamma_{n}\left(k_{z}, r\right)=i \rho_{0} c k \frac{R_{n}\left(k_{r} r\right)}{k_{r} R_{n}^{\prime}\left(k_{r} r_{S}\right)} .
$$

The dependency on $k_{z}$ stems from Eq. (6).

The radial functions describe a discrete spectrum in the angular domain but are continuous in the $k_{z}$-domain. Comparing the model description at hand to the interrelations of Fourier Transform and Fourier Series, this is not at all surprising.

\section{B. Properties}

Figure 3 shows an example of $\Gamma_{n}$ for $r=2 \mathrm{~m}, r_{S}=$ $0.15 \mathrm{~m}, \kappa=\pi$ and $f=5 \mathrm{kHz}$. To help with legibility,

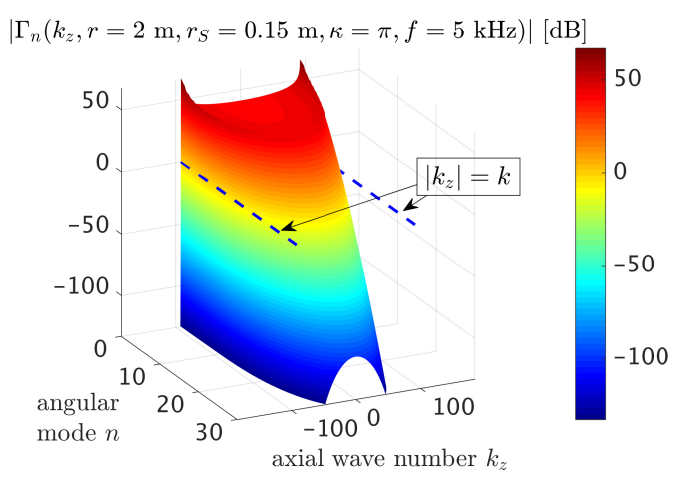

FIG. 3: (colour online) Magnitude of the radial functions $\Gamma_{n}\left(k_{z}, r\right)$ in $\mathrm{dB}$ (referred to unity), $r=2 \mathrm{~m}$, $r_{S}=0.15 \mathrm{~m}, \kappa=\pi, f=5 \mathrm{kHz}$.

the plot is continuous along the $n$-axis, but only discrete points of $n$ are relevant. It can be observed that modal combinations $\left(n, k_{z}\right)$ with axial wave numbers $k_{z}>k$ are heavily attenuated, i.e. they decay as evanescent wave components. The same applies for evanescent angular modes $n>k \frac{r_{S} \kappa}{\pi}$, only their descent is not as steep as for the axial wave numbers [2].

The radial functions describe the physics of the propagation inside wedge spaces: While purely axial modes $(n=0)$ cannot transition from evanescent into propagating modes as the radius increases, purely angular modes $\left(k_{z}=0\right)$ can. The reason is simply that a mode $n$ with a certain trace wavelength at a given radius may be evanescent, so that across a small arc section it 'shuffles' air between its maxima and minima, while at a larger radius, the same mode now has a longer trace wavelength and propagates, no longer effecting an acoustic 'short circuit'. This principle does not hold for axial modes, but it does for modal combinations $\left(n, k_{z}\right)$. With view on the $\mathrm{NAH}$ performance prospect, this finding suggests that having a small standoff distance, i.e. the gap between hologram (measurement) plane and $V$, is less critical for the NAH resolution in the $\phi$-domain than for the resolution in the $z$-domain (see Section V). 


\section{Truncation and Limitation of the Solutions}

As a consequence of the propagating modes accumulating around the origin of the $n-k_{z}$-plane, the integration and summation in (18) can be limited and truncated, respectively, while maintaining a good approximation of the actual sound field, provided that $A_{n}\left(k_{z}\right)$ has a similar, finite magnitude for all combinations $\left(n, k_{z}\right)$.

In the case of (18), the integration limit $K_{z}>k$ and the final term of the truncated sum $n=N$ can be chosen such that

$$
\tilde{p}(\mathbf{r})=\sum_{n=0}^{N} \int_{-K_{z}}^{K_{z}} \Gamma_{n}\left(k_{z}, r\right) V_{n}\left(k_{z}, r_{S}\right) \Phi_{n}(\phi) Z\left(k_{z} z\right) d k_{z}
$$

approximates the actual $p(\mathbf{r})$ adequately by monitoring the convergence of the integral and sum. This requires however the a priori knowledge of all coefficients $V_{n}\left(k_{z}, r_{H}\right)$ to determine the contribution of evanescent modes in comparison to the propagating modes.

Not only is the evaluation of the radial functions useful for the numerical calculation of sound fields, but they also provide valuable information when attempting to solve inverse problems $[2,21]$. The latter are a key component of NAH, whose methods are adapted for infinite wedges in the subsequent section.

\section{HOLOGRAPHY IN INFINITE WEDGES}

This section introduces the equations that enable acoustic holography, i.e. the reconstruction of pressure (and also particle velocity) at any point within the wedge space from the knowledge of a hologram $\{p(\mathbf{r})\}_{\mathbf{r} \in \Lambda}$ (see Section II C).

Equation (15) links pressure spectra obtained from different radii for infinite wedges. Replacing the expression for the pressure in Eq. (9) by Eq. (18) (evaluated at $r=r_{H}$ ) shows that the radial functions in (25) link the pressure spectra of the hologram at radius $r_{H}$ to the normal velocity spectra at the radius $r_{S}$, yielding

$$
P_{n}\left(k_{z}, r_{H}\right)=\Gamma_{n}\left(k_{z}, r_{H}\right) V_{n}\left(k_{z}, r_{S}\right) .
$$

A key component of NAH is to reformulate the equation as an expression to calculate the velocity spectrum, which can be obtained by multiplying both sides of the equation by the reciprocals of the radial functions. However, the evanescent nature of the higher order modes reflects in an extremely small magnitude of the radial functions for modes outside the "radiation circle" defined by $k=\sqrt{k_{z}^{2}+\left(\frac{\pi n}{\kappa r_{S}}\right)^{2}}$ (see Section IV B). Accordingly, their reciprocals take extremely large values, effecting heavy amplification of the corresponding pressure modes. While no problem in theory, the measurement noise of the hologram data in practice also manifests itself in the derived pressure spectra. When heavily amplified, the noise in the pressure spectrum can severely corrupt the result for $V_{n}\left(k_{z}, r_{S}\right)$. This common issue in the field of inverse problems can be addressed by introducing a Tikhonov regularisation to the reciprocal of the radial functions, however, there are alternatives that may provide better results in certain cases (see Section I). The regularised solutions for the velocity spectra $\tilde{V}_{n}\left(k_{z}, r_{S}\right)$ are then given by

$$
\tilde{V}_{n}\left(k_{z}, r_{S}\right)=\frac{\Gamma_{n}^{*}\left(k_{z}, r_{H}\right)}{\left|\Gamma_{n}\left(k_{z}, r_{H}\right)\right|^{2}+\beta_{V}^{2}} \hat{P}_{n}\left(k_{z}, r_{H}\right) .
$$

The $\hat{\imath}$ symbol on the pressure spectra denotes that they were calculated from holograms measured at $r_{H}$ by evaluating equation (9). A suitable regularisation parameter $\beta_{V}$ that is optimal for minimising the impact of noise in the measured data can be chosen through the L-Curve analysis $[41,47]$ or other techniques.

From the literature it is known that a Tikhonov regularisation of the inverse problem is equivalent to a spatial lowpass filtering of the synthesised sound field quantity (pressure and velocity). Examples of such surface velocity reconstructions from measured data and further practical aspects of the mechanisms described above are presented in the Section VIII.

Once the normal velocity spectrum has been obtained from (28), the velocity on $V$ can be reconstructed using the synthesis equation

$$
\tilde{v}_{r}\left(r_{S}, \phi, z\right)=\sum_{n=0}^{\infty} \int_{-\infty}^{\infty} \tilde{V}_{n}\left(k_{z}, r_{S}\right) \Phi_{n}(\phi) Z\left(k_{z} z\right) d k_{z}
$$

(compare to Eq. (10)).

\section{A. Considerations on the Practical Relevance}

With Equation (29) the radiation model is complete and theoretically all the mathematical expressions have been derived to perform acoustical holography from measured data.

In practice, however, the expressions in Eqs. (1), (10), (18) and even (26) require the uncountable set $\left\{Z\left(k_{z} z\right)\right\}_{k_{z} \in \mathbb{R}}$ to enable the description of any arbitrary pressure field. This is in analogy to the inverse Fourier Transform, mapping from the frequency domain to the time domain. An infinitely spreading propagation space however hardly applies to real life conditions; On the contrary, most of the time it is necessary to consider the sound field in a room with a floor at $z=z_{1}$ and a ceiling at $z=z_{2}$, i.e. there is an additional boundary condition for those values of $z$.

The following section introduces the key expressions that were derived for the infinite wedge in a form that pertains to the finite wedge instead, with a rigid or pressure release boundary condition at the floor and ceiling of the wedge. 


\section{RADIATION MODEL AND ACOUSTICAL HOLOGRAPHY FOR THE FINITE WEDGE}

The propagation space is now further restricted to a finite wedge by introducing two parallel planes $z=z_{1}$ (the floor) and $z=z_{2}$ (the ceiling), effecting a wedge-shaped wave guide. On top of the angular boundary conditions, the model should also satisfy the pressure release boundary condition, $\left.p(\mathbf{r})\right|_{z=z_{1}, z_{2}}=0$, or the rigid boundary condition, $\left.\frac{\partial}{\partial z} p(\mathbf{r})\right|_{z=z_{1}, z_{2}}=0$, respectively, on the floor $\left(z=z_{1}\right)$ and the ceiling $\left(z=z_{2}\right)$.

Following similar steps to those described in Sections II, III and V, the most important expressions for the radiation and acoustical holography have been derived and are presented in the remainder of this section.

\section{A. Pressure Radiated from the Boundary $V$ for Finite Wedges}

An expression similar to Eq. (18) that describes the radiation from the boundary

$$
V:=\left\{\mathbf{r}: r=r_{S}, \phi \in\left[\phi_{1}, \phi_{2}\right], z \in\left[z_{1}, z_{2}\right]\right\}
$$

is given through

$$
\begin{aligned}
p(\mathbf{r}) & =i \rho_{0} c k \sum_{n, m=0}^{\infty} \frac{R_{n}\left(k_{r}^{m} r\right)}{k_{r}^{m} R_{n}^{\prime}\left(k_{r}^{m} r_{S}\right)} V_{n}^{m}\left(r_{S}\right) \Phi_{n}(\phi) Z_{m}(z), \\
k_{r}^{m} & =\sqrt{k^{2}-\left(\frac{m \pi}{\zeta}\right)^{2}}, m \in \mathbb{N}_{0} .
\end{aligned}
$$

The new TOS of basis functions that satisfies the boundary conditions for the floor and ceiling is given through

$Z_{m}(z)=\mu_{m}\left\{\begin{array}{l}\sin \left(\frac{m \pi}{\zeta}\left(z-z_{1}\right)\right),(\text { Pressure Release BC) } \\ \cos \left(\frac{m \pi}{\zeta}\left(z-z_{1}\right)\right),(\text { Rigid BC })\end{array}\right.$

with $\mu_{m}=\sqrt{\frac{2-\delta_{m}}{\zeta}}$ and $\zeta=z_{2}-z_{1}$, where $z_{1}, z_{2} \in \mathbb{R}$, $z_{1}<z_{2}$ and $m \in \mathbb{N}_{0}$. This TOS is now countable, likewise yielding countable sets of radial and axial wave numbers, where the latter is given by $\left\{k_{z}^{m}\right\}_{m \in \mathbb{N}_{0}}:=$ $\left\{k_{z}^{m}=\frac{m \pi}{\zeta}: m \in \mathbb{N}_{0}\right\}$. In this case, both $\Phi_{n}(\phi) \in \mathbb{R}$ and $Z_{m}(z) \in \mathbb{R}$ do not describe propagating waves along the circumferential and axial direction, respectively, but standing waves instead. The transform from the spatial domain to the modal domain for the pressure is given through the double integral

$$
P_{n}^{m}(r)=\int_{\phi_{1}}^{\phi_{2}} \int_{z_{1}}^{z_{2}} p(r, \phi, z) \Phi_{n}(\phi) Z_{m}(z) d z d \phi .
$$

When transforming the normal velocity, $p(r, \phi, z)$ becomes $v(r, \phi, z)$ and $P_{n}^{m}(r)$ becomes $V_{n}^{m}(r)$, respectively.

One major advantage of the expression in (30) over the one in (18) is that it does not require (numerical) integration. The summations should be infinite, however, the series truncation error can be kept below a reasonable limit since the higher order components of $\Phi_{n}(\phi)$ and $Z_{m}(z)$ become evanescent [2] when $\frac{n \pi}{\kappa}>k_{r}^{m} r$. In that case the contribution of these particular components may be negligible, depending on the observation radius $r$ (see Section IV).

\section{B. Neumann-Green Function and Rayleigh-like Formula for Finite Wedges}

Following a similar procedure as presented in Section III yields the expression for the NGF in finite wedges

$$
\begin{aligned}
G_{N}\left(\mathbf{r} \mid \mathbf{r}^{\prime}\right)=\sum_{n, m=0}^{\infty} & \frac{1}{r_{S}} \frac{R_{n}\left(k_{r}^{m} r\right)}{k_{r}^{m} R_{n}^{\prime}\left(k_{r}^{m} r_{S}\right)} \\
& \cdot \Phi_{n}\left(\phi^{\prime}\right) Z_{m}\left(z^{\prime}\right) \Phi_{n}(\phi) Z_{m}(z)
\end{aligned}
$$

that likewise satisfies the boundary condition in Eq. (24) everywhere but at the position of the singularity/point source $\mathbf{r}^{\prime}=\left(r_{S}, \phi^{\prime}, z^{\prime}\right)^{T}$. The Rayleigh-like formula for the finite wedge is then given by

$$
p(\mathbf{r})=i \rho_{0} c k \int_{\phi_{1}}^{\phi_{2}} \int_{z_{1}}^{z_{2}} G_{N}\left(\mathbf{r} \mid \mathbf{r}^{\prime}\right) v_{n}\left(\mathbf{r}^{\prime}\right) r_{S} d z^{\prime} d \phi^{\prime} .
$$

\section{Nearfield Acoustical Holography in Finite Wedges}

The synthesis equation for the velocity reconstruction from the corresponding spectrum is given through

$$
\tilde{v}_{r}\left(r_{S}, \phi, z\right)=\sum_{n, m=0}^{\infty} \tilde{V}_{n}^{m}\left(r_{S}\right) \Phi_{n}(\phi) Z_{m}(z),
$$

where the velocity spectrum is calculated through

$$
\tilde{V}_{n}^{m}\left(r_{S}\right)=\frac{\Gamma_{n}^{*}\left(\frac{m \pi}{\zeta}, r_{H}\right)}{\left|\Gamma_{n}\left(\frac{m \pi}{\zeta}, r_{H}\right)\right|^{2}+\beta_{V}^{2}} \hat{P}_{n}^{m}\left(r_{H}\right)
$$

and the pressure spectrum is obtained from the measured hologram $\hat{p}\left(r_{H}, \phi, z\right)$ through

$$
\hat{P}_{n}^{m}\left(r_{H}\right)=\int_{\phi_{1}}^{\phi_{2}} \int_{z_{1}}^{z_{2}} \hat{p}\left(r_{H}, \phi, z\right) \Phi_{n}(\phi) Z_{m}(z) d z d \phi .
$$

\section{SOUND FIELD SIMULATIONS}

The numerical calculation of the pressure field was accomplished on the basis of the truncated synthesis Equa- 


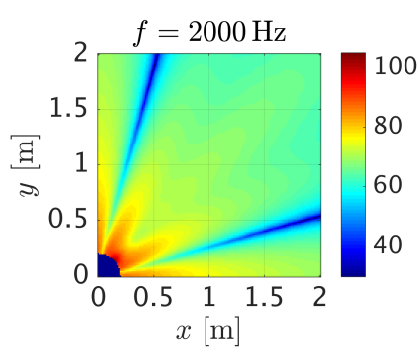

(a) Infinite Wedge

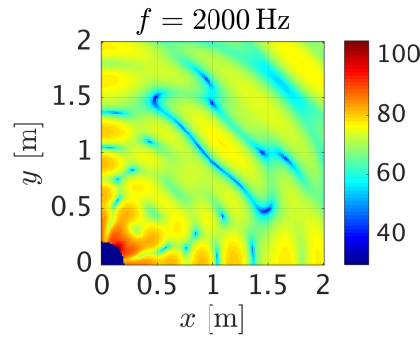

(b) Finite Wedge
FIG. 4: (colour online) Pressure field in $\mathrm{dB}$ (referred to unity) of a point source located at $\left(0.2, \frac{\pi}{4}, 0\right)^{T}, Q=1.5$,

$$
\Delta k_{z}=0.01
$$

tion (26), for the infinite wedge, and the correspondingly truncated expression in Equation (30) for the finite wedge. In the case of (26) it is additionally necessary to numerically solve the integration. For the results in this work, the truncated integration was numerically solved using the trapezoidal rule [48] ( $1^{\text {st }}$ order approximation) with the integration limits $K_{z}=\left[\frac{Q k}{\Delta k_{z}}\right] \Delta k_{z}, \quad Q \in \mathbb{R} \geq$ 1 , and the step size $\Delta k_{z}$ for the integration variable, where $\lceil\cdot\rceil$ denotes the ceiling operator. The ceiling operator ensures that all propagating wave components are definitely part of the synthesis. The parameter $Q$ can be adjusted to control the amount of evanescent components included in the calculation. Alternatively, the integral in (26) can be solved using the inverse Fast Fourier transform (IFFT) [2, 32], which is commonly done in the field of NAH as it may increase the speed of the calculation. The limits for the truncation of the sums over the angular and axial components were chosen as $N=\left\lceil Q k r_{S}\right\rceil$ and $M=\left\lceil\frac{Q k \zeta}{\pi}\right\rceil$, respectively.

The following subsections introduce two elementary types of sources mounted on the baffle for both propagation spaces.

\section{A. Point Source Model}

The NGFs presented in Sections III and VIB can be applied to model the sound field of a point source on the rigid baffle $V$ located at $\left(r_{S}, \phi^{\prime}, z^{\prime}\right)^{T}$ for the infinite and the finite wedge, respectively. The resulting pressure field can be calculated from Eq. (21) and Eq. (35), respectively, where

$$
v_{n}\left(\mathbf{r}^{\prime}\right)=v_{r}\left(\mathbf{r}^{\prime}\right)=\frac{1}{r_{S}} \delta\left(r-r_{S}\right) \delta\left(\phi-\phi^{\prime}\right) \delta\left(z-z^{\prime}\right) .
$$

Figure 4a shows the magnitude of the pressure field in the $x y$-plane $(z=0)$ for an infinite wedge with $\kappa=\frac{\pi}{2}$, $r_{S}=0.2 \mathrm{~m}, \phi^{\prime}=\frac{\pi}{4}$ and $z^{\prime}=0$. The interference between the radiated waves and the reflections from the rigid walls generate a clear radiation pattern. Particularly two angles at which destructive interference occurs point distinct nulls in the radiation pattern.
The same scenario for a finite wedge with $\kappa=\frac{\pi}{2}$ and $\zeta=2 \mathrm{~m}$, where $z_{1}=-1 \mathrm{~m}$ and $z_{2}=1 \mathrm{~m}$, is shown in Fig. 4b. In comparison to the field shown in Fig. 4a, the field in the finite wedge also suggests a weakened radiation in those same directions where Fig. 4a shows distinct destructive interference, the attenuation however being nowhere near as strong. This must be due to the now occurring reflections from the floor and ceiling that overlay their own interference pattern. The latter is responsible for increased maxima and minima observed when increasing the radius along the angle $\phi=\phi^{\prime}$.

\section{B. Piston Source Model}

Another type of source that is simple to simulate with the given model is that of a piston source on the baffle. It can be modelled as a patch in the radial component of the velocity profile on the boundary $V$ where $v_{r}\left(r_{S}, \phi^{\prime}, z^{\prime}\right) \neq$ 0 . For a patch located centrally on $V$, of angular width $\alpha$ and axial width $B$, the velocity profile is given by the product

$$
v_{r}\left(r_{S}, \phi, z\right)=\Pi\left(\frac{\phi-\frac{\kappa}{2}}{\alpha}\right) \Pi\left(\frac{z}{B}\right)
$$

where $\Pi$ denotes the rectangular function

$$
\Pi(\phi)=\left\{\begin{array}{l}
1,|\phi| \leq \frac{1}{2}, \\
0,|\phi|>\frac{1}{2} .
\end{array}\right.
$$

The corresponding radial component of the velocity spectra for the infinite and finite wedge, as calculated from (12) and (33) (see Section VI A), are

$$
\begin{array}{r}
V_{n}\left(k_{z}, r_{S}\right)=\alpha \\
\operatorname{sinc}\left(k_{z} \frac{B}{2}\right) Z^{*}\left(k_{z} 0\right) \\
\cdot \operatorname{sinc}\left(\frac{n \pi}{\kappa} \frac{\alpha}{2}\right) \Phi_{n}\left(\frac{\pi}{4}\right)
\end{array}
$$

and

$$
\begin{aligned}
& V_{n}^{m}\left(r_{S}\right)=\alpha B \operatorname{sinc}\left(\frac{m \pi}{\zeta} \frac{B}{2}\right) Z_{m}\left(\frac{\zeta}{2}\right) \\
& \cdot \operatorname{sinc}\left(\frac{n \pi}{\kappa} \frac{\alpha}{2}\right) \Phi_{n}\left(\frac{\pi}{4}\right),
\end{aligned}
$$

respectively. In this case Eqs. (18) and (30), respectively, are more suitable to directly calculate the sound fields. The results for the infinite and finite wedge are shown in Figures 5a and 5b, respectively. With the piston size in a similar order of magnitude as the wave length, the fields in Fig. 5 show similar characteristics as those in Fig. 4a, yet the attenuation with propagated distance is smaller than that of the point source. The overall level difference between the results for the point source and those for the piston source stems from the leading factor $\alpha B$ in Eqs. (42) and (43). 


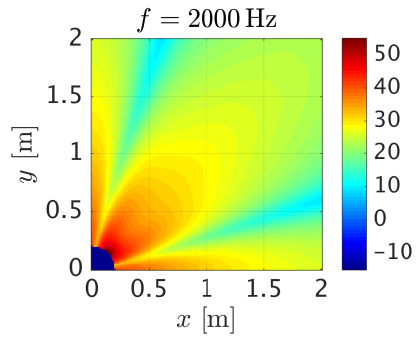

(a) Infinite Wedge

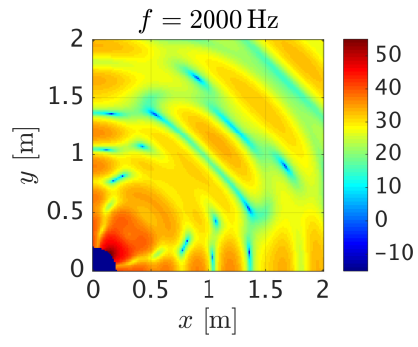

(b) Finite Wedge
FIG. 5: (colour online) Pressure field in $\mathrm{dB}$ (referred to unity) of a piston located at $\left(0.2, \frac{\pi}{4}, 0\right)^{T}, Q=1.5$, $\Delta k_{z}=0.01, \alpha=\frac{\pi}{6}, B=0.1 \mathrm{~m}$.

\section{MEASUREMENTS}

In order to support the developed theory and model, holographic measurements of piston-like sources were conducted inside a quasi-infinite wedge with $\kappa=\pi$. The prototype of a hemi-cylindrical loudspeaker array [49] with 15 Tang Band W1-1070SH drivers was used as a radiating source with $r_{S}=0.1426 \mathrm{~m}$. The measured hologram data is compared to simulated data, assuming the sound field of rectangular pistons with an edge length equal to the diameter of the drivers in the prototype, each located in the corresponding positions. Pressure spectra were calculated from the measured holograms in order to perform NAH. The reconstructed velocity profile of the radiating surface is then compared to the underlying actual source profile.

The next subsection introduces the measurement rig in detail.

\section{A. Measurement Rig}

The prototype was placed on a large planar baffle inside an anechoic chamber (see Fig. 6). Absorbing mate-

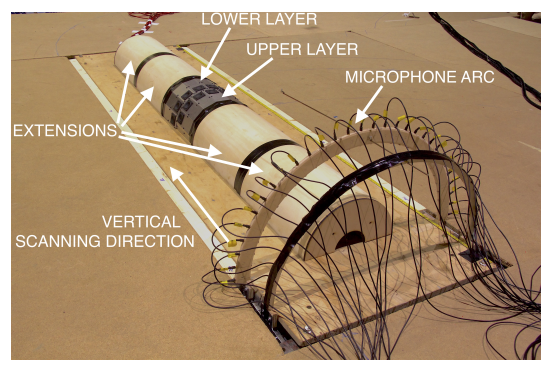

FIG. 6: (colour online) Measurement rig with an array of 30 G.R.A.S. 40PL array microphones fitted on an arch over the HCA with radius $r_{H}=0.3 \mathrm{~m}$.

rial was placed at the edges of the planar baffle to reduce the reflections caused by the locally occurring change of acoustic impedance. The prototype was extended with wooden elements to locally provide conditions similar to those of an infinite hemi-cylinder.

Two sliding rails were fitted underneath the baffle, running parallel to the axis of the HCA on either side. Each held two carriers: one pair to support a wooden arc with radius $r_{H}=0.3 \mathrm{~m}$, holding 30 G.R.A.S. $40 P L$ array microphones (see Fig. 6) and one pair to hold a strain relief feeding the microphone cables to the array. This arrangement minimises the acoustic obstruction posed by the measurement arc and allows for iterative scanning of the sound field radiated from the HCA along the vertical axis. The gaps in the baffle exposing the two rails were covered with pieces of timber to mitigate the acoustic impedance change occurring at the gap and thereby reducing reflections.

\section{B. Angular Sampling and Resolution}

The $S_{\phi}=30$ microphones were arranged on the arc at the angles $\phi_{u}=\frac{\pi}{S_{\phi}}\left(u-\frac{1}{2}\right), u=1 \ldots S_{\phi}$, yielding a uniform sampling pattern. Given the nature of the angular basis functions $\Phi_{n}(\phi)$ with the fixed phase relation, it follows from the sampling theorem that this microphone arrangement allows for the recovery of orders up to $n=N_{M}=S_{\phi}-1=29$. However, Fig. 3 suggests that, up to a certain frequency, high angular orders will have already decayed below the noise floor once they have propagated to the measurement aperture given their evanescent nature. To investigate this further, one can look at the magnitude of the integral over the axial component of the radial functions

$$
\Gamma_{n}^{(C)}\left(r, r_{S}, \kappa\right)=\int_{-K_{z}}^{K_{z}} \Gamma_{n}\left(k_{z}, r, r_{S}, \kappa\right) d k_{z}
$$

as a function of both radius and frequency. Figure 7 shows the magnitude of $\Gamma_{n}^{(C)}$ normalised to the modal strength at $r=r_{S}$. This result suggests it is unlikely that orders $n \geq 12$ can be observed above the noise floor when they reach the measurement aperture at $r_{H}=0.3 \mathrm{~m}$ for frequencies below $10 \mathrm{kHz}$.

\section{Vertical Sampling and Resolution}

The measured holograms were obtained at $z \in$ $[-0.5,0.5] \mathrm{m}$, covering $A_{Z}=1 \mathrm{~m}$ with $S_{Z}=51$ samples. The sampling positions along the $z$-axis are $z_{w}=$ $\Delta z\left(w-\frac{S_{Z}+1}{2}\right), w=1 \ldots S_{Z}$. It follows from the sampling theorem that the vertical sample spacing enables capture of axial components $k_{z}$ up to $k_{z, \max }=\frac{\pi}{\Delta z} \approx$ $157.1 \frac{\mathrm{rad}}{\mathrm{m}}$. Due to the finite hologram aperture along the $z$-axis, a spatial 8-point Tukey window [2] is applied to the sampled data to attenuate otherwise salient higher spatial frequency components, which would occur due to the discontinuity at the edges of the hologram. The hologram is then extended to an aperture length ranging 

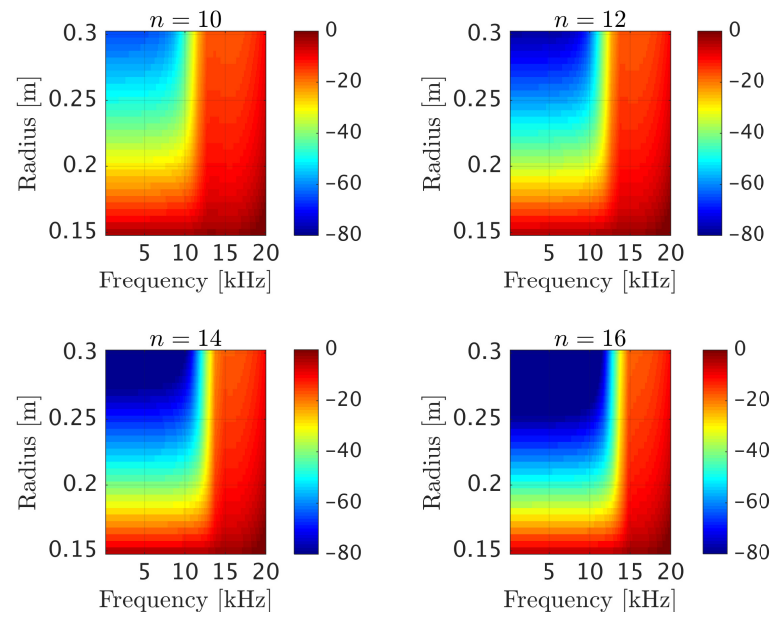

FIG. 7: (colour online) Modal decay in dB from source surface $\left(r_{S}=0.1426 \mathrm{~m}\right)$ to hologram surface

$\left(r_{H}=0.3 \mathrm{~m}\right)$ computed from $\Gamma_{N}^{(C)}$ within the audible frequency band. The data is normalised to the modal strength on the surface of the loudspeaker array.

from $-3 \mathrm{~m}$ to $3 \mathrm{~m}$ by zero-padding, so that $A_{Z}=6 \mathrm{~m}$ and $S_{Z}=301$.

\section{Analysis Equations}

The analysis equation for the finite measurement aperture and set of samples is given by

$$
\hat{P}_{n}\left(k_{z}, r_{H}\right)=\frac{\kappa}{S_{\phi}} \sum_{u=1}^{S_{\phi}} \Phi_{n}\left(\phi_{u}\right) \Delta z \sum_{w=1}^{S_{Z}} Z^{*}\left(k_{z} z_{w}\right) \hat{p}\left(\phi_{u}, z_{w}\right),
$$

where $\hat{p}\left(\phi_{u}, z_{w}\right)$ is the pressure measured at the $u$ th microphone at the height $z_{w}$ at the hologram radius $r_{H}=0.3 \mathrm{~m}$, where the spatial window has already been applied. Similar to the frequency domain resolution of the Discrete Time Fourier Transform (DTFT), the pressure spectrum $\hat{P}_{n}\left(k_{z}, r_{S}\right)$ can be obtained at an arbitrary resolution in the $k_{z}$-domain from the measurement data $\hat{p}\left(\phi_{u}, z_{w}\right)$. For the acquired hologram, the analysis was performed with a resolution of $\Delta k_{z}=\frac{2 k_{z, \max }}{S_{z}}$, matching that of a standard FFT operation.

\section{E. Reconstruction Error Measure}

The difference between velocity reconstructions obtained from the measured hologram and a simulated reference is quantified through the Normalised Mean Squared Reconstruction Error (NMSRE) on the recon- struction aperture, given through

$$
\mathrm{NMSRE}=10 \log _{10}\left\{\frac{\sum_{u^{\prime}=1}^{S_{\phi}} \sum_{w^{\prime}=1}^{S_{Z}}\left|e\left(\phi_{u^{\prime}}, z_{w^{\prime}}\right)\right|^{2}}{\sum_{u^{\prime}=1}^{S_{\phi}} \sum_{w^{\prime}=1}^{S_{Z}}\left|v_{r}\left(\phi_{u^{\prime}}, z_{w^{\prime}}\right)\right|^{2}}\right\},
$$

with $e\left(\phi_{u^{\prime}}, z_{w^{\prime}}\right)=\tilde{v}_{r}\left(\phi_{u^{\prime}}, z_{w^{\prime}}\right)-v_{r}\left(\phi_{u^{\prime}}, z_{w^{\prime}}\right)$, where $v_{r}\left(\phi_{u^{\prime}}, z_{w^{\prime}}\right)$ denotes the velocity reference obtained through holographic reconstruction from simulated data acquired on the extended measurement aperture to avoid zero-padding. The reconstruction aperture consists of 201 samples in the $\phi$-domain and 401 samples in the $z$ domain in the interval $[-0.5,0.5] \mathrm{m}$, each uniformly distributed.

\section{F. Result for a Single Driver}

The field radiated from a driver/piston located at $\left(r_{S}, \frac{\pi}{2}, 0.0315 \mathrm{~m}\right)^{T}$ is considered. The measured hologram is compared against one hologram obtained from perturbed and one from ideal simulation. The perturbed data is created from the model by introducing normally distributed positioning errors with a standard deviation of $4 \mathrm{~mm}$ in $z$-direction and $2 \mathrm{~mm}$ in $\phi$-direction to the measurement points and by adding noise to the calculated data. The Signal-to-Noise-Ratio (SNR) was set so that it matches that of the measured data, which was estimated on the basis of noise samples from the tail of the measured impulse responses as suggested in [50].

The measured hologram was not absolutely calibrated, so any given pressure data does NOT represent the actual SPL. The measured and the simulated ideal holograms are put into perspective by normalising the former to the latter at the hologram point closest to the driver.

Figure 8a shows the magnitude and phase of the different holograms at $f=8 \mathrm{kHz}$. Both magnitude and phase show the effects of reflections from the walls at the angles $\phi=0$ and $\phi=\pi$. Comparing the measured and the perturbed simulation data to the ideal model data, it can be observed that the non-ideal holograms show artefacts due to the various acquisition perturbations.

Figure 8b shows the magnitude of the pressure spectra $\hat{P}_{n}\left(k_{z}, r_{H}\right)$ of the non-ideal holograms. Due to the symmetry of the setup, the spectrum of the ideal hologram would be expected not to contain any contributions at odd angular orders $n$. However, measurement noise and positioning errors of the measurement rig and prototype contribute to an intrinsic noise floor in the spectra, which adds to the prominent contributions at even orders $n$.

Using the pressure spectra $\hat{P}_{n}\left(k_{z}, r_{H}\right)$, the radial component of the velocity spectrum on the boundary of the prototype $\left(r_{S}=0.1426 \mathrm{~m}\right)$ can be obtained from Eq. (28) and numerical evaluation of Eq. (29). It was observed from the work with the measured data that the exact knowledge of hologram radius $r_{H}$ and baffle radius $r_{S}$ is critical for accurate reconstruction of the spreading of the radiation source along the $\mathrm{z}$-axis. 

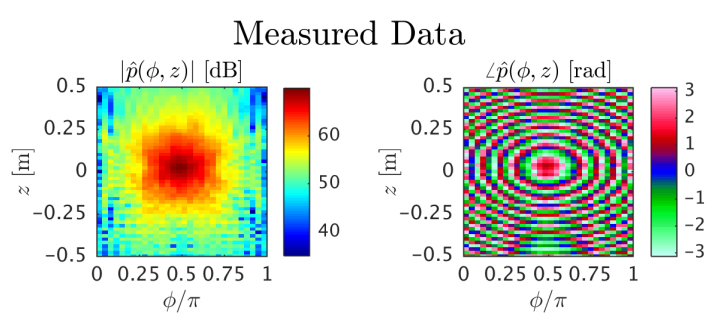

Perturbed Simulation Data
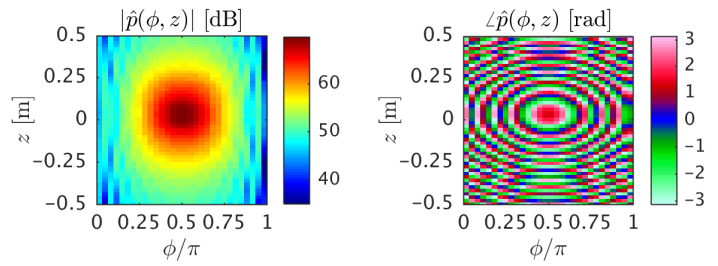

Ideal Simulation Data
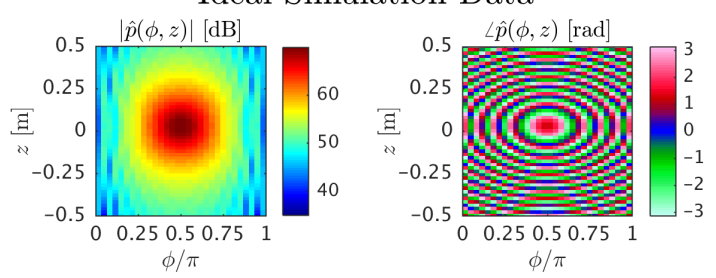

(a) Hologram Magnitude Data (in dB, referred to unity)
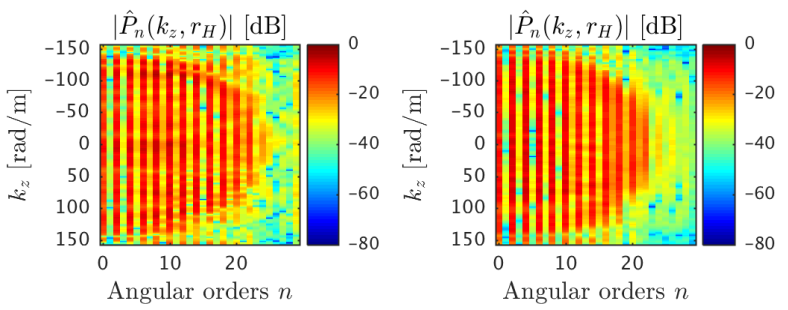

(b) Spatial Pressure Spectra (in dB, referred to unity) of (left) measured and (right) perturbed simulation data

FIG. 8: (colour online) (a) Magnitude and phase of holograms for a driver at $\left(r_{S}, \frac{\pi}{2}, 0.0315 \mathrm{~m}\right)^{T}, f=8 \mathrm{kHz}$.

The holographic radial velocity reconstruction in Fig. 9a shows a very clear maximum of $v_{r}$ for all three types of data at the position of the driver. Note that the $\phi$-axis was translated into a position $x$ along the arc to ensure an undistorted image of the diaphragm, where $x(\phi)=r_{S} \phi, \phi \in[0, \kappa]$. The reference reconstruction is obtained from the ideal simulated hologram on the extended measurement aperture after using the same regularised inverse filter as for the measured hologram. Compared to the reference, the reconstruction from both measured and perturbed simulation data shows artefacts that must be attributed to measurement noise and position errors.

Figure $9 \mathrm{~b}$ shows the corresponding magnitude spectra in the modal domain. The lowpass-filter effect of the Tikhonov regularisation can be clearly observed when comparing the velocity spectra to the pressure spectra of the hologram data in Fig. 8b. The background noise at high values of $n$ and $\left|k_{z}\right|$ is suppressed by the regularisation.
Reconstr. from Measured Data
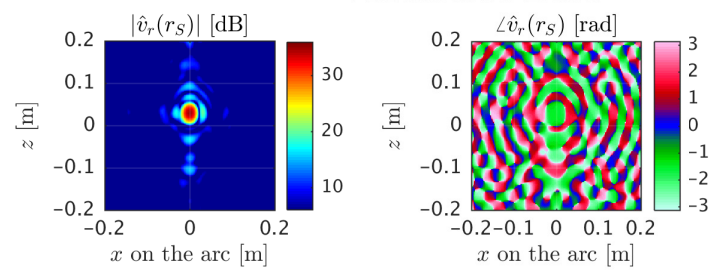

Reconstr. from Perturbed Sim. Data
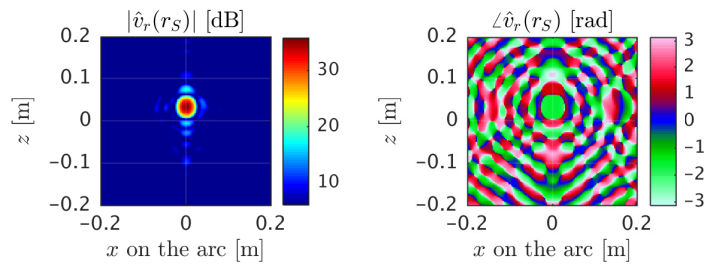

Reconstr. from Sim. Data
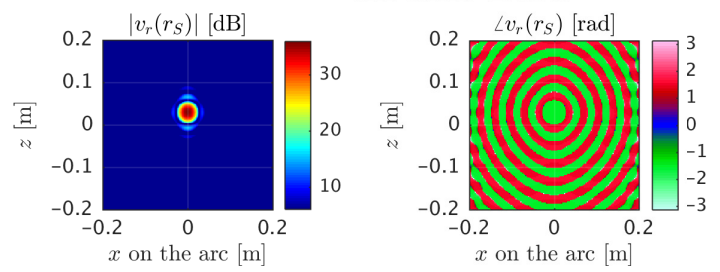

(a) Velocity Reconstruction (in $\mathrm{dB}$, referred to unity)
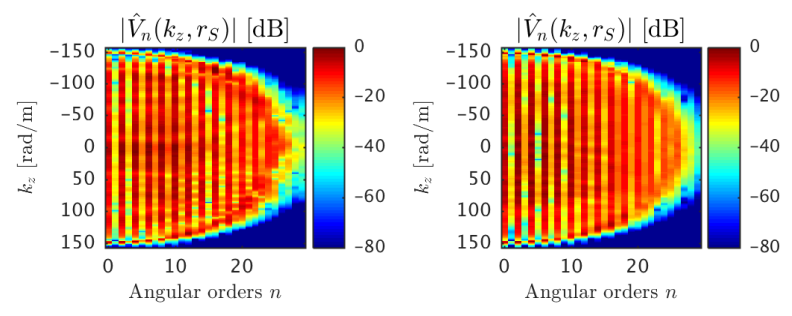

(b) Velocity Spectra (in dB, referred to unity) from (left) measured and (right) perturbed simulation data.

FIG. 9: (colour online) (a) Holographic velocity reconstruction on the prototype surface, (b) corresponding magnitude spectra.

To ascertain the influence of the measurement perturbations on the NMSRE, the reconstruction from the measured hologram is compared against results obtained with perturbed simulation holograms in the frequency range from $1 \mathrm{kHz}$ to $8.5 \mathrm{kHz}$. The NMSRE for both cases is shown in Fig. 10. The results based on perturbed sim-

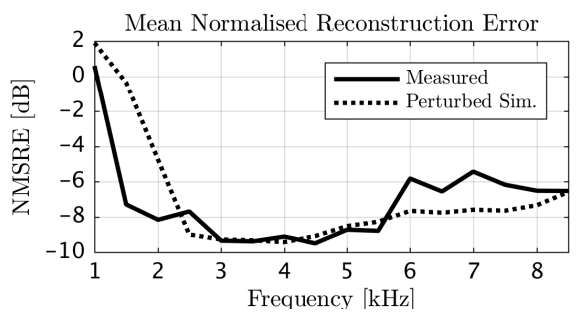

FIG. 10: NMSRE of holographic reconstruction from measured and perturbed sim. data, $1 \mathrm{kHz}$ to $8.5 \mathrm{kHz}$. 
ulated holograms are averaged over 20 samples for each frequency. It can be seen that, with a difference mostly smaller than $2 \mathrm{~dB}$, the NMSRE results are of very similar magnitude and development for both types of data.

\section{G. Discussion}

From the presented hologram data, it can be seen that there is generally a good match between the measurements and simulations, especially considering that the piston shape of measurement and simulation is slightly different. Physical effects such as the reflections from the rigid baffles at $\phi=0$ and $\phi=\pi$ can be observed in all holograms, as expected from Section VII.

The results of the holographic velocity reconstructions show an overall convincing match with the reference. It can be seen that the extent of the area indicating the piston position is not perfectly round but looks prolonged in $z$-direction. This supports the hypothesis made in Section IV B, since the spatial resolution (given the standoff distance) is sufficient to clearly distinguish the driver's diaphragm from the side lobes in the $\phi$-domain yet not in the $z$-domain.

Other reconstruction artefacts might be attributed to measurement noise and positioning errors (see below), as well as a relatively short scanning aperture and a large standoff distance; A smaller hologram radius $r_{H}$ would provide a better SNR and require less regularisation, thus increasing the spatial resolution of the velocity reconstruction at the baffle radius $r_{S}$ while reducing the side lobes. There is, however, a tradeoff since decreasing $r_{H}$ shifts spatial aliasing effects to lower frequencies as higher order wave components are stronger near the source. Furthermore, it should also be noted that the reconstruction of the velocity from a pressure hologram is conditioned worse than the reconstruction from a velocity hologram, as described by Jacobsen and Liu in [51]. This exacerbates the influence of noise and requires more regularisation, effecting a lower reconstruction resolution.

Comparing the NMSRE of the holographic reconstructions from measured and perturbed simulation data suggests that the biggest part of the reconstruction error can, with good confidence, be attributed to imperfections in the measurement rig and the prototype, e.g. positioning errors, the relatively short aperture and noise. The difference in NMSRE may also occur due to the effects of reflections from sudden impedance changes (discontinuities of the baffle, the scanning arc and the trenches housing the supporting rails) that contribute to the measured data but cannot easily be modelled. This result suggests that the discrepancy between the model and the measured data is dominated by imperfections of the measurement rig and the SNR and implies a good accuracy of the model for the given geometry.

\section{SUMMARY \& OUTLOOK}

Acoustic models for the propagation of waves and radiation from sources in infinite and finite wedge-shaped propagation spaces have been presented. From a basic expansion through orthonormal basis functions proposed for both types of propagation spaces, Rayleigh-like formulae as well as corresponding NGFs were derived from a basic expansion. These have been shown to provide simulated data that are well-matched with hologram data obtained from measurements. The identification of radial functions from the Rayleigh-like formulae enabled the formulation of a set of forward and inverse propagators that were then applied to perform NAH on the basis of measured hologram data. Using the proposed NAH method, reconstructions of the normal velocity on a hemi-cylindrical baffle obtained from measured and simulated holograms were presented to confirm both the developed sound field model and its NAH suitability. A criterion for truncation of the model's series and integrals has been proposed.

It was found from the measurement results that a smaller standoff distance would produce more accurate results. The spatial resolution that remained after regularisation reduced the accuracy of the velocity sources along the vertical axis by comparison to the actual driver layout. A hypothesis suggesting that the angular resolution is less sensitive to an inadequate choice for the standoff distance than the axial resolution was proposed and confirmed from the measurement data.

\section{ACKNOWLEDGMENTS}

This work has been partially funded by HUAWEI. E.G. Williams is supported by the US Office of Naval Research.
[1] M. J. Buckingham, "Hybrid formulation of wave propagation and scattering," (Springer Netherlands, Dor- drecht, 1984) Chap. Acoustic Propagation in a WedgeShaped Ocean with Perfectly Reflecting Boundaries, pp. 
77-99.

[2] E. G. Williams, Fourier Acoustic: Sound Radiation and Nearfield Acoustical Holography (Academic Press, 24-28 Oval Road, London NW1 7DX, UK, 1999) pp. 1-295.

[3] J. Meyer, "Beamforming for a circular microphone array mounted on spherically shaped objects," Journal of the Acoustical Society of America 109, 185-193 (2001).

[4] T. D. Abhayapala and D. B. Ward, "Theory and design of high order sound field microphones using spherical microphone array," in 2002 IEEE International Conference on Acoustics, Speech, and Signal Processing, Vol. 2 (2002) pp. 1949-1952.

[5] J. Meyer and G. W. Elko, "A highly scalable spherical microphone array based on an orthonormal decomposition of the soundfield," in 2002 IEEE International Conference on Acoustics, Speech, and Signal Processing (IEEE, 2002) pp. 1781-1784.

[6] Z. Li, R. Duraiswami, E. Grassi, and L. S. Davis, "Flexible layout and optimal cancellation of the orthonormality error for spherical microphone arrays," in 2004 IEEE International Conference on Acoustics, Speech, and Signal Processing (2004) pp. 1520-6149.

[7] S. Moreau, J. Daniel, and S. Bertet, "3D sound field recording with higher order Ambisonics - objective measurements and validation of a 4 th order spherical microphone," in Preprints of the 120th AES Convention (2006).

[8] H. Teutsch and W. Kellermann, "Acoustic source detection and localization based on wavefield decomposition using circular microphone arrays," Journal of the Acoustical Society of America 120, 2724-2736 (2006).

[9] W. Luo and R. Zhang, "Exact solution of threedimensional acoustic field in a wedge with perfectly reflecting boundaries," Science China Physics, Mechanics \& Astronomy 58, 1-10 (2015).

[10] J. Daniel, S. Moreau, and R. Nicol, "Further investigations of high-order ambisonics and wavefield synthesis for holophonic sound imaging," in 114th Convention of the Audio Engineering Society (2003).

[11] J. Ahrens and S. Spors, "An analytical approach to sound field reproduction using circular and spherical loudspeaker distributions," Acta Acustica united with Acustica 94, 988-999 (2008).

[12] F. M. Fazi, M. Noisternig, and O. Warusfel, "Representation of sound fields for audio recording and reproduction," in Acoustics 2012: 11ème Congrès Français d'Acoustique. Annual meeting of the Institute of Acoustics, Nantes, FR, 23 - 27 Apr 2012 (2012).

[13] J. Ahrens, Analytic Methods of Sound Field Synthesis, T-Labs Series in Telecommunication Services (Springer Verlag, Berlin Heidelberg, 2012) pp. 1-272.

[14] P. N. Samarasinghe, T. D. Abhayapala, and M. A. Poletti, "Room reflections assisted spatial sound field reproduction," in Proceedings of the 22nd European Signal Processing Conference (EUSIPCO), 2014 (2014) pp. 1352-1356.

[15] G. Weinreich and E. B. Arnold, "Method for measuring acoustic radiation fields," The Journal of the Acoustical Society of America 68, 404-411 (1980).

[16] J. D. Maynard, E. G. Williams, and Y. Lee, "Nearfield acoustic holography: I. theory of generalized holography and the development of NAH," Journal of the Acoustical Society of America 78, 1395-1413 (1985).

[17] Y. T. Cho, J. S. Bolton, and J. Hald, "Source visual- ization by using statistically optimized near-field acoustical holography in cylindrical coordinates," The Journal of the Acoustical Society of America 118, 2355-2364 (2005).

[18] E. G. Williams, N. Valdivia, P. C. Herdic, and J. Klos, "Volumetric acoustic vector intensity imager," Journal of the Acoustical Society of America 120, 1887-1897 (2006).

[19] E. G. Williams and K. Takashima, "Vector intensity reconstructions in a volume surrounding a rigid spherical microphone array," The Journal of the Acoustical Society of America 127, 773-783 (2010).

[20] F. Jacobsen, G. Moreno-Pescador, E. Fernandez-Grande, and J. Hald, "Near field acoustic holography with microphones on a rigid sphere (1)," The Journal of the Acoustical Society of America 129, 3461-3464 (2011).

[21] D. Colton and R. Kress, Inverse Acoustic and Electromagnetic Scattering Theory (Springer New York Heidelberg Dordrecht London, 1998) pp. 1-405.

[22] F. M. Fazi, Sound Field Reproduction, Ph.D. thesis, University of Southampton, Faculty of Engineering, Science and Mathematics, Institute of Sound and Vibration Research (2010).

[23] M. Kolundzija, C. Faller, and M. Vetterli, "Baffled circular loudspeaker array with broadband high directivity," in Proceedings of the IEEE International Conference on Acoustics, Speech, and Signal Processing, ICASSP 2010, 14-19 March 2010, Sheraton Dallas Hotel, Dallas, Texas, USA (2010) pp. 73-76.

[24] M. Kolundzija, C. Faller, and M. Vetterli, "Design of a compact cylindrical loudspeaker array for spatial sound reproduction," in 130th Convention of the Audio Engineering Society (Audio Engineering Society, 2011).

[25] A.-G. Webster, "Acoustical impedance, and the theory of horns and of the phonograph," Proceedings of the National Academy of Science 5, 275-282 (1919).

[26] J. Blauert and N. Xiang, Acoustics for Engineers (Springer Berlin Heidelberg, 2008) pp. 1-218.

[27] J. B. Allen and D. A. Berkley, "Image method for efficiently simulating small-room acoustics," Journal of the Acoustical Society of America 65, 943-950 (1979).

[28] L. E. Kinsler, A. R. Frey, A. B. Coppens, and J. V. Sanders, Fundamentals of Acoustics, 4th ed., edited by B. Russiello (John Wiley \& Sons, Inc., New York Chichester Weinheim Brisbane Singapore Toronto, 2000) pp. 1-548.

[29] M. J. Buckingham, "Theory of acoustic radiation in corners with homogeneous and mixed perfectly reflecting boundaries," Journal of the Acoustical Society of America 86, 2273-2291 (1989).

[30] J. S. Asvestas, J. J. Bowman, P. L. Christiansen, O. Einarsson, R. E. Kleinman, D. L. Sengupta, T. B. A. Senior, F. B. Sleator, P. L. E. Uslenghi, and N. R. Zitron, Electromagnetic and Acoustic Scattering by Simple Shapes, edited by J. J. Bowman, T. B. A. Senior, and P. L. E. Uslenghi (North-Holland Pub. Co. - Amsterdam, 1970) pp. 252-282.

[31] E. G. Williams, "Regularization methods for near-field acoustical holography," Journal of the Acoustical Society of America 110, 1976-1988 (2001).

[32] E. G. Williams, B. H. Houston, and P. C. Herdic, "Fast fourier transform and singular value decomposition formulations for patch nearfield acoustical holography," Journal of the Acoustical Society of America 114, 13221333 (2003). 
[33] G. H. Koopmann, L. Song, and J. B. Fahnline, "A method for computing acoustic fields based on the principle of wave superposition," The Journal of the Acoustical Society of America 86, 2433-2438 (1989).

[34] Z. Wang and S. F. Wu, "Helmholtz equation-leastsquares method for reconstructing the acoustic pressure field," The Journal of the Acoustical Society of America 102, 2020-2032 (1997).

[35] A. Schuhmacher, J. Hald, K. B. Rasmussen, and P. C. Hansen, "Sound source reconstruction using inverse boundary element calculations," The Journal of the Acoustical Society of America 113, 114-127 (2003).

[36] F. Jacobsen and V. Jaud, "Statistically optimized near field acoustic holography using an array of pressurevelocity probes," The Journal of the Acoustical Society of America 121, 1550-1558 (2007).

[37] Y.-B. Zhang, F. Jacobsen, C.-X. Bi, and X.-Z. Chen, "Near field acoustic holography based on the equivalent source method and pressure-velocity transducers," The Journal of the Acoustical Society of America 126, 12571263 (2009).

[38] M. R. Bai, C. Wang, and S.-W. Juan, "Optimal two-layer directive microphone array with application in near-field acoustical holography," The Journal of the Acoustical Society of America 132, 862-871 (2012).

[39] J. Hald, "Fast wideband acoustical holography," The Journal of the Acoustical Society of America 139, 15081517 (2016).

[40] W. Klippel and C. Bellmann, "Holographic nearfield measurement of loudspeaker directivity," in 141st Convention of the Audio Engineering Society (2016).

[41] P. C. Hansen, Rank-Deficient and Discrete IllPosed Problems (Society for Industrial and Applied Mathematics, 3600 University City Science Center, Philadelphia, PA 19104-2688, USA, 1998) pp. 1-247, http://epubs.siam.org/doi/pdf/10.1137/1.9780898719697.

[42] F. M. Fazi and P. A. Nelson, "The ill-conditioning problem in sound field reconstruction," in 123rd Convention of the Audio Engineering Society (2007).

[43] G. Chardon, L. Daudet, A. Peillot, F. Ollivier, N. Bertin, and R. Gribonval, "Near-field acoustic holography using sparse regularization and compressive sampling principles," The Journal of the Acoustical Society of America 132, 1521-1534 (2012).

[44] J. Antoni, "Synthetic aperture acoustical holography," in PROCEEDINGS OF ISMA2012-USD2012 (2012) pp. 3525-3538.

[45] E. Kreyszig, Introductory Functional Analysis with Application (Wiley, 1978) pp. 1-688.

[46] A. Cruz and J. Sesma, "Zeros of the Hankel function of real order and of its derivative," Mathematics of Computation 39, 639-645 (1982).

[47] P. C. Hansen, "The L-curve and its use in the numerical treatment of inverse problems," in in Computational Inverse Problems in Electrocardiology, ed. P. Johnston, Advances in Computational Bioengineering (WIT Press, 2000) pp. 119-142.

[48] I. N. Bronstein, K. A. Semendjajew, G. Musiol, and H. Mühlig, Taschenbuch der Mathematik (Handbook of Mathematics) (Wissenschaftlicher Verlag Harri Deutsch GmbH, Frankfurt am Main, Germany, 2006) pp. 926-927.

[49] F.-M. Hoffmann, F. M. Fazi, and S. Fontana, "Sound field control with hemi-cylindrical loudspeaker arrays," in Audio Engineering Society Conference: 2016 AES In- ternational Conference on Sound Field Control (2016).

[50] G.-B. Stan, J. J. Embrechts, and D. Archambeau, "Comparison of different impulse response measurement techniques," Journal of the Audio Engineering Society 50, 249-262 (2002).

[51] F. Jacobsen and Y. Liu, "Near field acoustic holography with particle velocity transducers," The Journal of the Acoustical Society of America 118, 3139-3144 (2005). 